\title{
Improving the mental health of Roma through research and policies that cross multiple social sectors
}

\author{
Benjamin Lê Cook $^{1,2} \cdot$ Ana Progovac ${ }^{1,2} \cdot$ Nathaniel Tran ${ }^{1}$
}

Received: 15 May 2019/Accepted: 16 May 2019/Published online: 30 May 2019

(C) Swiss School of Public Health (SSPH+) 2019

Roma face ongoing discrimination and social exclusion resulting in deleterious consequences to their mental health, despite being one of the largest ethnic minorities in Europe (Foldes and Covaci 2012). Yet, there is little to no systematic research that guides approaches to improving mental health among the Roma (Cook et al. 2013).

Roma are at a higher risk of mental illness because they are more likely to lack access to the opportunities granted by income, education, employment, and safe and stable housing, factors compounded by unfair social policies and individual experiences of discrimination (Sandor et al. 2017). Roma often face policies that exclude them from public spaces or deny them access to hygienic and safe housing, or even subject them to forced eviction or deportation. This leads to separation of families, erosion of cultural identity, and Roma distrust of housing authorities, with subsequent effects on mental health and well-being (Cemlyn et al. 2009). Second, Roma youth are often excluded from and/or have experienced discrimination within the educational system, leading to a cycle of poor school achievement or dropout, unemployment, and housing instability, all factors having strong associations with psychological stress and depression (Greenfields and Smith 2010). These cycles of instability in the presence of overlapping social risk factors can interact to become unmanageable for individuals or systems alike and can heighten mental health risks. On an interpersonal level, experiences of discrimination "get under the skin," leading to increased cortisol and other stress hormones that accelerate disease processes, poorer health behaviors (substance use, smoking, risky sexual behaviors), and increased psychological distress and depressive symptoms (Pascoe and Richman 2009).

Benjamin Lê Cook

bcook@cha.harvard.edu

1 Health Equity Research Lab, Cambridge Health Alliance, 1035 Cambridge St., Suite 26, Cambridge, MA 02141, USA

2 Department of Psychiatry, Harvard Medical School, Boston, MA, USA
Despite this multitude of risk factors, there have been few surveys with structured psychiatric diagnostic instruments among the Roma. One of the few examples is a survey in Serbia that found that Roma were more frequently unhappy and unsatisfied with their life compared to non-Roma (Cvjetkovic et al. 2017). Similarly, Roma children surveyed in Romania and Bulgaria experienced more poverty, stigma, and discrimination than non-Roma children, resulting in a higher burden of mental health problems (Lee et al. 2014). Among the uninsured in Portugal, Roma had significantly poorer mental health driven in part by a higher number of depression, anxiety, and psychotic symptoms (Pereira et al. 2016). Another study found that Roma experience rates of anxiety, depression, suicidal ideation, and suicide attempt that are many times greater than the population average (Cemlyn et al. 2009). This scant evidence generally finds worse mental health among Roma, but there is a clear need for more rigorous and generalizable psychiatric epidemiological data.

Improving mental health among Roma will also require a systematic approach to implementing interventions across multiple levels, including macro level policies addressing system-level factors (housing, education, healthcare access), and family and individual-level needs. Non-medical social determinants of mental health (education, employment, housing, and healthcare) were the target of the European Commission's National Roma Integration Strategies. However, evaluations of these initiatives have been mixed: some countries improved access to services like tap water and electricity, while in other countries, discrimination toward Roma in access to housing may actually have increased, with inconsistent compliance and enforcement from government entities across EU and non-EU countries (Lau and Ridge 2011). Similarly, until very recently, anti-racism campaigns that challenge societal norms have rarely focused on discrimination against Roma, which often goes unchecked in the media, leading to feelings that the Roma culture and way of life is not valued or respected. In response, Roma sometimes choose not to identify as Roma, or identify as another 
ethnic group in order to avoid racism (Cemlyn et al. 2009). Falling short on these policy initiatives has direct consequences on the mental health of the Roma.

Policy reform, descriptive research studies, and evaluation of adapted, evidence-based interventions are needed. Policy Reform: Roma integration policy initiatives that directly impact the mental health of the Roma need to be reinstituted and enforced. The EU Framework on mental health and well-being should specifically recommend tailoring evidence-based interventions for vulnerable populations. Descriptive research studies: More rigorous psychiatric epidemiology studies are needed with structured diagnostic instruments, and measures of social exclusion and social determinants such as housing and healthcare. Roma communities themselves should be engaged in establishing priorities for these descriptive studies. Evidence-based interventions: In one of the few examples of an intervention that tracks mental health of the Roma, qualitative data from a Hungarian intervention evaluation suggests that relocation of families from camps into permanent housing led to improved mental health, decreased infectious disease, ill health, disability, and mortality (Molnár et al. 2011). The extent to which relocation interventions would be appropriate and acceptable to Roma with varying cultural backgrounds and differing desires to live in settled communities is unknown and should be further investigated. Interventions that provide psychoeducation, treatment, and/or awareness of the impact of discrimination and racism on mental health are needed. Use of trusted Roma community members called mediators (Schaaf et al. 2016) who can connect other members of their community with service providers is an approach that could be expanded for improving mental health among the Roma. Rollout of mental health interventions should blend strong community engagement with research methods that carefully evaluate the adaptation and implementation of evidence-based interventions.

\section{Compliance with ethical standards}

Conflict of interest The authors declare that they have no conflict of interest.

\section{References}

Cemlyn S, Greenfields M, Burnett S, Matthews Z, Whitwell C (2009) Inequalities experienced by Gypsy and traveller communities: a review. Equality and Human Rights Commission Research Report Series (Winter 2009)

Cook B, Wayne GF, Valentine A, Lessios A, Yeh E (2013) Revisiting the evidence on health and health care disparities among the Roma: a systematic review 2003-2012. Int J Public Health 58(6):885-911. https://doi.org/10.1007/s00038-013-0518-6

Cvjetkovic S, Jankovic J, Bjegovic-Mikanovic V (2017) Differences in predictors of Subjective Well-Being among Roma and NonRoma young adults in Serbia: Smiljana Cvjetkovic. Eur J Public Health, 27(Supplement 3):374-375

Foldes ME, Covaci A (2012) Research on Roma health and access to healthcare: state of the art and future challenges. Int J Public Health 57(1):37-39. https://doi.org/10.1007/s00038-011-0312-2

Greenfields M, Smith DM (2010) Housed Gypsy Travellers, social segregation and the reconstruction of communities. Hous Stud 25(3):397-412

Lau AY-H, Ridge M (2011) Addressing the impact of social exclusion on mental health in Gypsy, Roma, and Traveller communities. Ment Health Soc Incl 15(3):129-137

Lee EJ, Keyes K, Bitfoi A, Mihova Z, Pez O, Yoon E, Masfety VK (2014) Mental health disparities between Roma and non-Roma children in Romania and Bulgaria. BMC Psychiatry 14(1):297

Molnár Á, Ádám B, Antova T, Bosak L, Dimitrov P, Mileva H, Pekarcikova J, Zurlyte I, Gulis G, Ádány R, Kósa K, Kósa K (2011) Health impact assessment of Roma housing policies in Central and Eastern Europe: a comparative analysis. Environ Impact Assess Rev 33:7-12

Pascoe E, Richman LS (2009) Perceived discrimination and health: a meta-analytic review. Psychol Bull 135(4):531-554

Pereira H, Amaral H, Monteiro S, Esgalhado G, Afonso RM, Loureiro M (2016) Mental health disparities between Roma and NonRoma unemployment insurance beneficiaries in Portugal. Diversidad and equality in Health and Care 13(3):246-250

Sandor J, Kosa Z, Boruzs K, Boros J, Tokaji I, McKee M, Adany R (2017) The decade of Roma Inclusion: did it make a difference to health and use of health care services? Int J Public Health 62(7):803-815. https://doi.org/10.1007/s00038-017-0954-9

Schaaf M, Pana A, Ileanu B, Metodieva M (2016) The costs and benefits of Roma Health Mediation in Bulgaria and Romania. Eur J Public Health 26(suppl_1):ckw174-105

Publisher's Note Springer Nature remains neutral with regard to jurisdictional claims in published maps and institutional affiliations. 